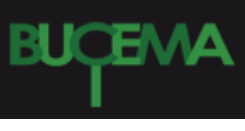

Bulletin du centre d'études médiévales d'Auxerre | BUCEMA

$19.1 \mid 2015$

Varia

\title{
Une clé pour l'approche du phénomène urbain au haut Moyen Âge à Troyes (Aube) : l'étude du mobilier archéologique
}

Mémoire de master 2, sous la direction de Denis Cailleaux, université de Bourgogne, et de Michel Kasprzyk, Inrap. Soutenu en juin 2013.

\section{Claire Bourguignon}

\section{(2) OpenEdition}

\section{Journals}

Electronic version

URL: https://journals.openedition.org/cem/13984

DOI: $10.4000 /$ cem. 13984

ISSN: 1954-3093

\section{Publisher}

Centre d'études médiévales Saint-Germain d'Auxerre

\section{Electronic reference}

Claire Bourguignon, "Une clé pour l'approche du phénomène urbain au haut Moyen Âge à Troyes

(Aube) : l'étude du mobilier archéologique", Bulletin du centre d'études médiévales d'Auxerre / BUCEMA

[Online], 19.1 | 2015, Online since 29 June 2015, connection on 22 September 2022. URL: http:// journals.openedition.org/cem/13984; DOI: https://doi.org/10.4000/cem.13984

This text was automatically generated on 22 September 2022.

Creative Commons - Attribution-NonCommercial-ShareAlike 4.0 International - CC BY-NC-SA 4.0 https://creativecommons.org/licenses/by-nc-sa/4.0/ 


\section{Une clé pour l'approche du phénomène urbain au haut Moyen Âge à Troyes (Aube) : l'étude du mobilier archéologique}

Mémoire de master 2, sous la direction de Denis Cailleaux, université de Bourgogne, et de Michel Kasprzyk, Inrap. Soutenu en juin 2013.

\section{Claire Bourguignon}

1 L'historiographie sur le fait urbain au haut Moyen Âge en Gaule septentrionale a longtemps réduit la ville à un espace en déclin, placé sous l'autorité religieuse. Durant cette période, l'espace urbain connaît pourtant d'importantes mutations de l'occupation $\mathrm{du}$ sol et de l'architecture ${ }^{1}$. Les fouilles menées dans

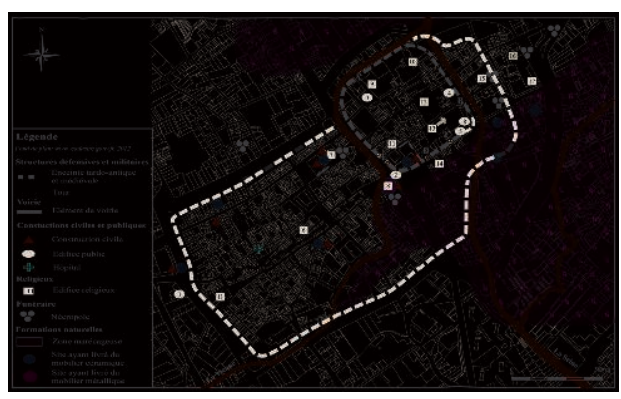
les centres urbains, tels Paris ou Lyon, ont mis en évidence le maintien des politiques édilitaires - construction d'édifices religieux et de palais comtaux - et ont suggéré un changement dans la conception de l'espace héritée de l'Antiquité.

2 Ce mémoire s'inscrit dans la continuité des travaux réalisés en archéologie urbaine médiévale jusqu'au début des années 2010, en proposant une approche de l'urbanisation de Troyes. Cette cité champenoise, située au sud-est du Bassin parisien, dans la plaine alluviale de la Seine (fig. 1), a fait l'objet d'un bilan documentaire concernant l'évolution de sa topographie du $\mathrm{I}^{\mathrm{er}}$ au XII ${ }^{\mathrm{e}}$ siècle. 
Fig. 1 - Localisation de la ville de Troyes et du site d'habitat rural du haut Moyen Âge de Fontvannes/Les Tomelles dans le département de l'Aube en Champagne-Ardenne

(CAO C. Bourguignon, 2014).

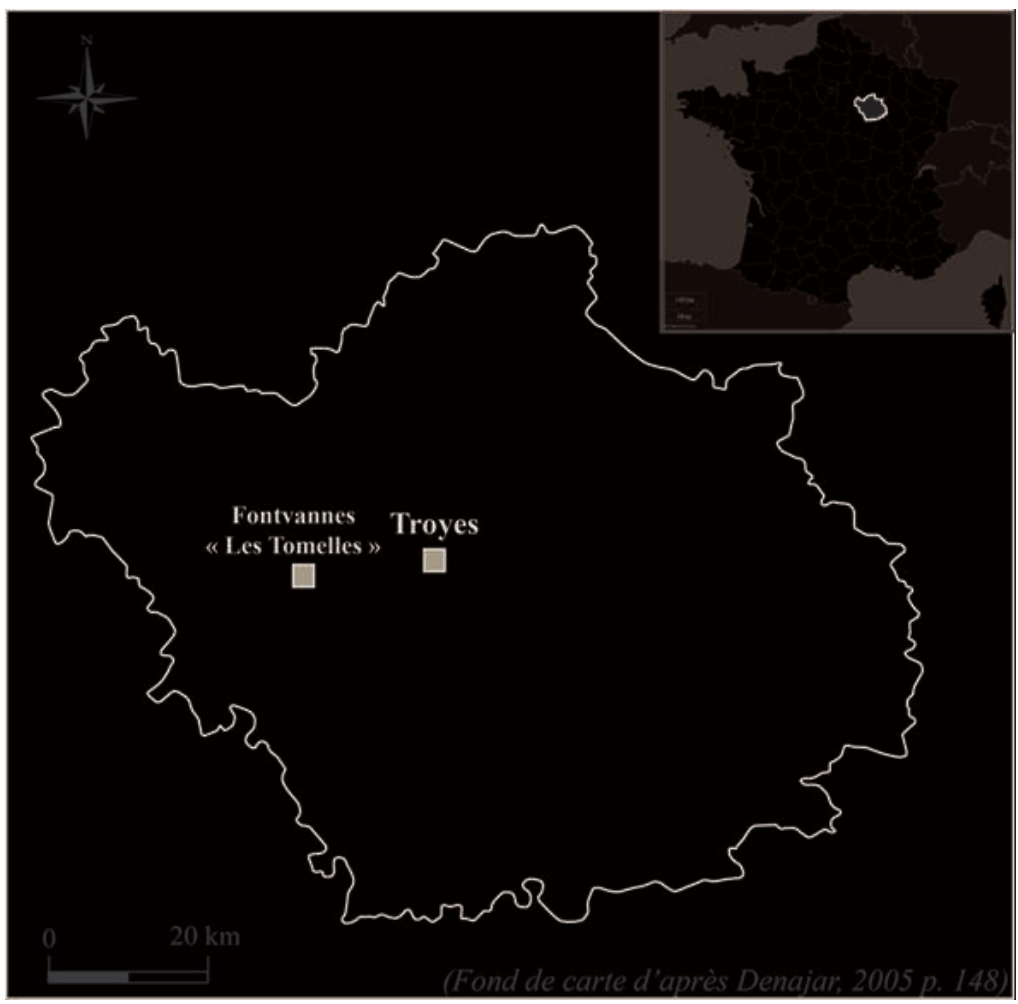

Ce travail est fondé sur un réexamen de la bibliographie antérieure et sur une nouvelle étude du fait urbain à la lumière des découvertes effectuées depuis les années $1990^{2}$. Ces recherches ont suggéré un développement de l'occupation au haut Moyen Âge audelà de l'espace clos par une enceinte, vraisemblablement construite à la fin du $\mathrm{III}^{\mathrm{e}}$ ou au début $d u \mathrm{IV}^{\mathrm{e}}$ siècle apr. J.-C. Cette hypothèse semblait infirmer l'idée d'un déclin de la ville au cours de cette période. Il a donc paru judicieux d'approfondir la réflexion en concentrant la recherche sur le mobilier trouvé au sein de l'espace urbain du haut Moyen Âge ${ }^{3}$. Cette analyse a pu bénéficier des avancées de la recherche (notamment typologiques) sur le mobilier de cette période ${ }^{4}$ et a permis de compléter les études sur la ville médiévale ${ }^{5}$.

4 Les travaux concernant Troyes aux périodes historiques débutent au XVIII siècle (fig. 2). 
Fig. 2 - Répartition des opérations concernant le haut Moyen Âge à Troyes (Aube), XIX siècle-2013 (CAO C. Bourguignon, 2015 ; fond de plan : www.cadastre.gouv.fr).

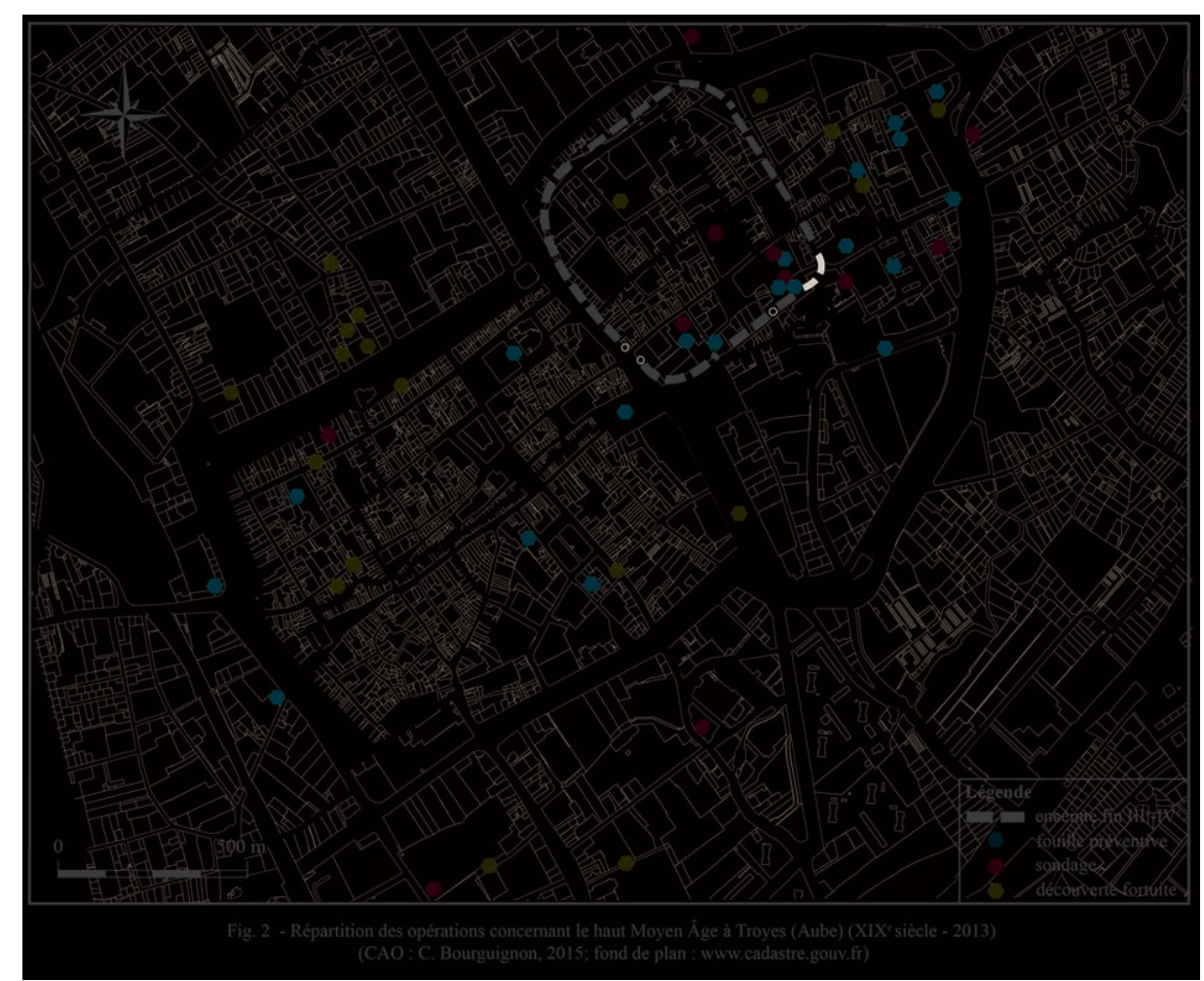

Les premiers vestiges découverts sont répertoriés en $1783^{6}$. Au XIX ${ }^{\mathrm{e}}$ siècle, les découvertes se multiplient. Parallèlement, plusieurs études portant sur la capitale auboise ${ }^{7}$ et le comté de Champagne au Moyen Âge ${ }^{8}$ sont publiées. Les travaux d'aménagements urbains des années 1960-1970 entraînent la découverte de vestiges essentiels à la connaissance du passé médiéval de Troyes. Depuis les années 1980, avec le développement de l'archéologie urbaine, les opérations se sont multipliées et la recherche s'est progressivement structurée. Un programme de recherche sur la cité et sa périphérie proche a été mis en place ${ }^{9}$. Deux ouvrages ont réalisé la synthèse des données, connues notamment pour le haut Moyen Âge : le Document d'évaluation du potentiel archéologique urbain ${ }^{10}$ et une thèse de doctorat portant sur la vie chrétienne dans le diocèse de Troyes $d u I^{e}$ au IX ${ }^{e}$ siècle ${ }^{11}$. Ces publications n'accordent qu'une place restreinte au mobilier, car l'essentiel des études de mobilier du haut Moyen Âge effectuées dans le cadre d'opérations archéologiques n'est pas publié.

6 Pourtant, l'examen du mobilier permet d'acquérir certaines données - durée d'occupation d'un secteur, passage de l'homme à une époque donnée... - non renseignées par les structures bâties mises au jour. C'est pourquoi l'objectif est de montrer en quoi l'étude d'un corpus de mobilier céramique et métallique constitue un potentiel informatif important dans l'étude des modes et des lieux de vie des populations urbaines au haut Moyen Âge.

7 Le corpus est composé de 1149 tessons de céramique potentiellement étudiables et 85 éléments de mobilier métallique (tab. 1 et 2) recueillis lors de quarante-trois interventions archéologiques (cf. fig. 2). 
Tab. 1 - Nombre de vases découverts à Troyes (Aube) par type de vases et par période. Seuls les vases dont la datation est possible ont été répertoriés ici (CAO C. Bourguignon, université de Bourgogne, 2013).

\begin{tabular}{|c|c|c|c|}
\hline \multirow{2}{*}{ Type de vase } & \multicolumn{3}{|l|}{ Nombre de vases par période } \\
\hline & 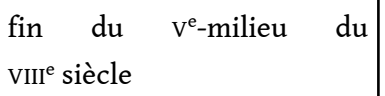 & $\begin{array}{l}\text { milieu du } \quad \text { VIII }^{\mathrm{e}}- \\
\mathrm{x}^{\mathrm{e}} \text { siècle }\end{array}$ & $\begin{array}{l}\mathrm{XI}^{\mathrm{e}} \text {-début } \\
\mathrm{XII}^{\mathrm{e}} \text { siècle }\end{array}$ \\
\hline \multicolumn{4}{|l|}{ Formes fermées : } \\
\hline $\begin{array}{l}\text { cruche, cruchon et } \\
\text { pichet }\end{array}$ & & 2 & 41 \\
\hline pot à cuire & & 4 & 10 \\
\hline \multicolumn{4}{|l|}{ Formes ouvertes : } \\
\hline bol & & 1 & \\
\hline \multicolumn{4}{|l|}{ écuelle } \\
\hline gobelet & 4 & & \\
\hline jatte & 1 & & \\
\hline pot & 1 & & \\
\hline
\end{tabular}

Tab. 2 - Nombre d'objets en métal découverts à Troyes (Aube) par domaines et catégories fonctionnelles et par période. Seuls les objets dont la datation est possible ont été répertoriés ici (CAO C. Bourguignon, université de Bourgogne, 2013).

\begin{tabular}{|c|c|c|c|}
\hline \multirow{2}{*}{$\begin{array}{l}\text { Domaines et catégories } \\
\text { fonctionnelles }\end{array}$} & \multicolumn{3}{|l|}{ Nombre d'objets par période } \\
\hline & $\begin{array}{l}\text { fin } \quad d u \quad v^{e}-\text { milieu } \quad d u \\
\text { VIII }^{\mathrm{e}} \text { siècle }\end{array}$ & $\mid \begin{array}{lll}\text { milieu } & d u & \text { VIII }^{\mathrm{e}}- \\
\mathrm{x}^{\mathrm{e}} \text { siècle } & & \end{array}$ & $\begin{array}{l}\mathrm{XI}^{\mathrm{e}} \text {-début } \\
\mathrm{XII}^{\mathrm{e}} \text { siècle }\end{array}$ \\
\hline \multicolumn{4}{|l|}{ Personnel: } \\
\hline parure, vêtement & 32 & & 1 \\
\hline \multicolumn{4}{|l|}{ Militaire : } \\
\hline équipement militaire & 15 & & \\
\hline \multicolumn{4}{|l|}{ Transport: } \\
\hline équipement lié à l'animal & 1 & & 12 \\
\hline \multicolumn{4}{|l|}{ Inclassable: } \\
\hline indéterminé & & & 5 \\
\hline
\end{tabular}




\begin{tabular}{|l|l|l|l|}
\hline divers, polyvalent & & & 2 \\
\hline Domestique : & & & \\
\hline activités culinaires & 4 & & 1 \\
\hline Immobilier : & & & \\
\hline huisserie & & & 1 \\
\hline Production: & & & \\
\hline divers, production & & & 1 \\
\hline
\end{tabular}

8 Le protocole d'étude du mobilier céramique s'appuie sur les méthodes et les typologies mises en ligne par le réseau Information sur la céramique médiévale et moderne (ICERAMM) au cours des années 2000 (http://iceramm.univ-tours.fr.). Les méthodes d'analyse du mobilier métallique utilisées reposent sur les travaux du groupe de recherche MétalInstrumentum publiés en $2013{ }^{12}$. Les données ont été enregistrées sous les logiciels Microsoft Excel et FileMakerPro. Les ruptures typologiques observées lors de l'étude du mobilier ont permis d'élaborer un découpage chronologique divisé en trois phases, qui semblent correspondre à des schémas différents d'évolution du fait urbain.

9 La première phase d'occupation correspond à la période courant de la fin du v $\mathrm{v}^{\mathrm{e}}$ siècle au milieu du viII siècle (fig. 3).

Fig. 3 - Troyes à la période mérovingienne, fin du ve siècle-milieu du ville siècle (CAO C. Bourguignon, 2014 ; fond de plan : DEBORDE et LENOBLE, 1995, pl. 6).

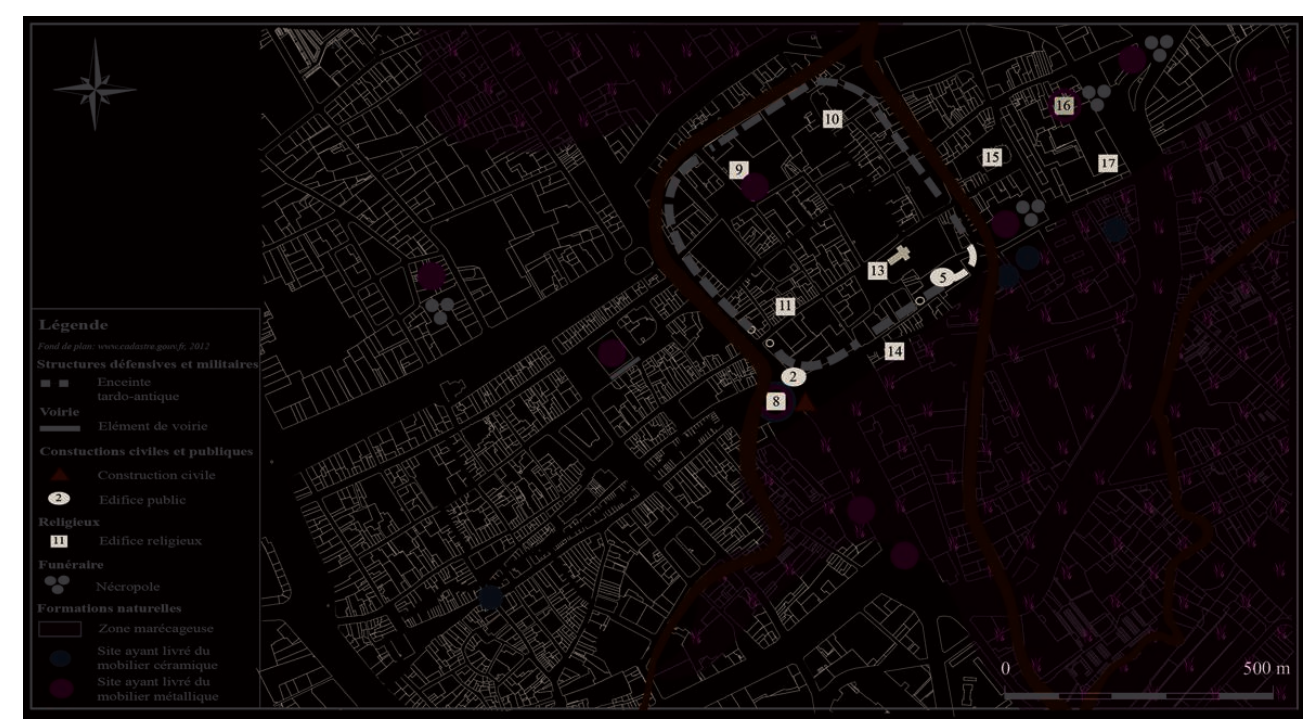

Les principaux éléments de mobilier céramique sont des fragments de pots à cuire et de bols datant des $\mathrm{VII}^{\mathrm{e}}$-VIII ${ }^{\mathrm{e}}$ siècles. Un fragment de cruche du viII ${ }^{\mathrm{e}}$ siècle est décoré à la molette (bandeau de losanges) - sites du 3-5, rue du Bon Pasteur ${ }^{13}$ et rue des Guillemets/Rue Breslay ${ }^{14}$. Ces découvertes témoignent d'une occupation du secteur oriental extra-muros aux abords du castrum. Le mobilier céramique découvert est typique de la période avec une prédominance des formes ouvertes (vases carénés, 
gobelets) et des décors à la molette (losanges, casiers). Le mobilier métallique relève de la parure: plusieurs fibules, dont une en bronze doré décorée de têtes d'oiseaux entourées de grenats (rue Surgale); des plaques-boucles, dont une en bronze damasquinée ornée d'un décor torsadé (rue Saint-Martin-ès-Aires). Ces vestiges ont été découverts à proximité immédiate de l'espace délimité par les églises Saint-Nizier ( $\mathrm{vI}^{\mathrm{e}}$ siècle), Saint-Aventin ( $\mathrm{VII}^{\mathrm{e}}$ siècle) et la première abbaye Saint-Loup ( $\mathrm{vi}^{\mathrm{e}}$ siècle). Ces églises, dont la fondation est uniquement connue par les textes médiévaux ${ }^{15}$, suggèrent la formation progressive d'un bourg ecclésiastique. Les données matérielles confirmeraient l'existence d'un foyer de peuplement, qui se développe aux $\mathrm{VI}^{\mathrm{e}}-\mathrm{VIII}^{\mathrm{e}}$ siècles ${ }^{16}$. L'emplacement des nécropoles à Saint-Martin-ès-Aires et dans l'avenue du Premier Mai, connu par les interventions archéologiques ${ }^{17}$, pourrait être lié à l'influence des établissements religieux nouvellement implantés à l'est. La proximité de l'enceinte tardo-antique à une centaine de mètres et la présence d'un élément de voirie extra-muros à l'est de l'espace urbain ${ }^{18}$ pourraient être des indices de l'insertion progressive de cet espace dans la dynamique des activités urbaines. En l'absence d'éléments plus probants, ces suppositions demeurent cependant à l'état d'hypothèses.

11 À l'ouest, dans un périmètre de 250 à $500 \mathrm{~m}$ de l'enceinte, l'occupation est principalement attestée par les découvertes de mobilier, dont une majorité a été trouvée en contexte funéraire : en particulier un fragment de gobelet tripartite à décor de casiers $\mathrm{du} \mathrm{VI}^{\mathrm{e}}$ siècle, découvert dans une sépulture implantée dans un vaste espace funéraire place de la Libération ${ }^{19}$, ou une agrafe à double crochet trouvée au chevet de l'église Saint-Rémy place des Halles ${ }^{20}$. Ce mobilier a été mis au jour dans des nécropoles, c'est-à-dire au sein d'espaces funéraires structurés. En l'absence de structures bâties à proximité, il n'est pas possible de déterminer leur articulation avec l'espace proche. Cette occupation devient plus dense dès la période suivante.

12 La deuxième phase d'occupation concerne la période s'étendant du milieu du $\mathrm{VIII}^{\mathrm{e}} \mathrm{au}$ $\mathrm{x}^{\mathrm{e}}$ siècle (fig. 4).

Fig. 4 - Troyes à la période carolingienne, milieu du VIII ${ }^{\mathrm{e}}$ siècle- $\mathrm{X}^{\mathrm{e}}$ siècle (CAO C. Bourguignon, 2014 ; fond de plan : DEBORDE et LENOBLE, 1995, pl. 6).

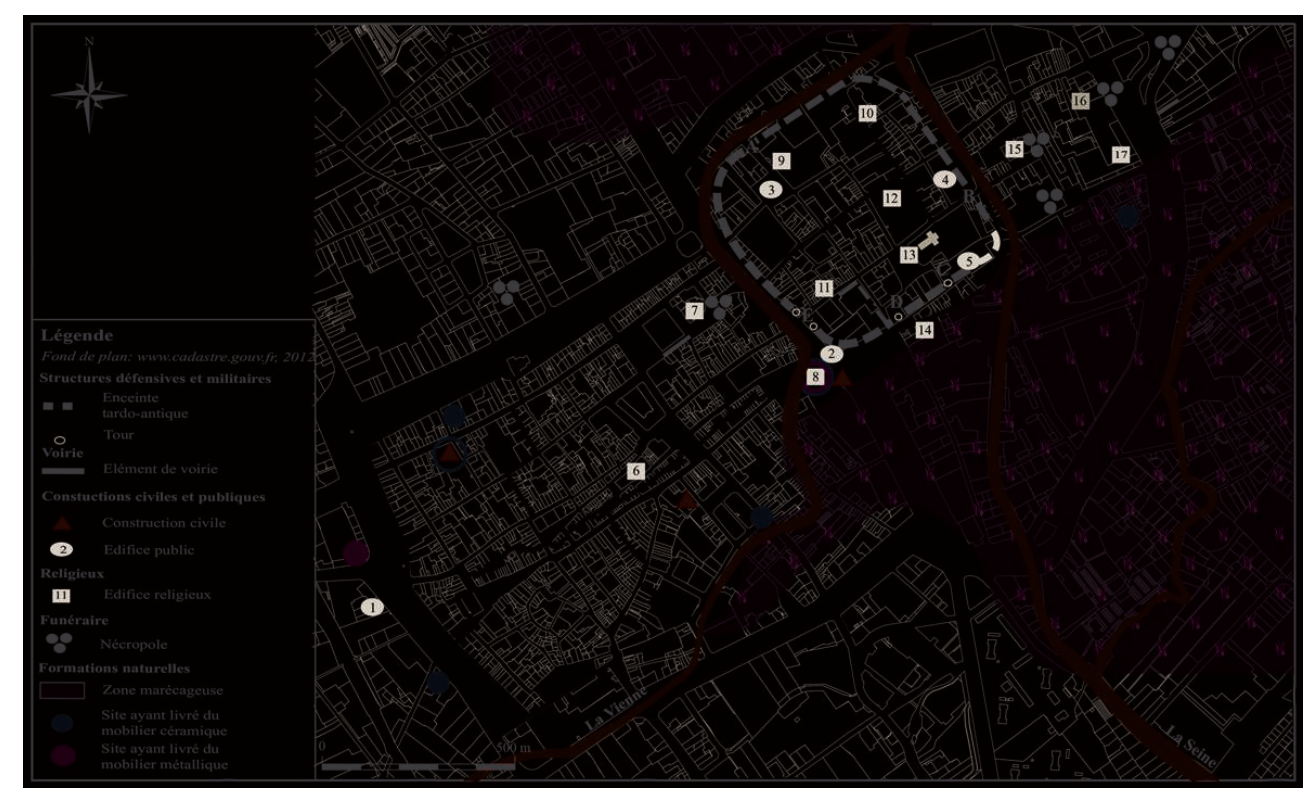

Bulletin du centre d'études médiévales d'Auxerre | BUCEMA, 19.1 | 2015 
L'essentiel des découvertes de mobilier concerne la partie sud-ouest extra-muros. Le mobilier céramique est constitué de vases globuleux à fond convexe spatulé - rue du Palais de Justice/rue du Général de Gaulle ${ }^{21}$ - et de fragments d'anses à rebords arrondis à paroi gris foncé - 130 , rue du Général de Gaulle/9, rue Argence ${ }^{22}$. Le mobilier céramique domestique se simplifie: les formes fermées (oules, pots à cuire) se développent, les récipients à pâte blanche prédominent. Les décors deviennent rares. Un étrier en fer de forme ovoïde du $\mathrm{x}^{\mathrm{e}}$ siècle, probablement d'origine hongroise, a aussi été trouvé place du Général Patton/rue Voltaire ${ }^{23}$. Ces découvertes sont intéressantes, car elles prouvent l'existence d'un foyer de peuplement qui n'est pas lié aux espaces funéraires. Or ce type d'occupation est déjà attesté aux $\mathrm{VI}^{\mathrm{e}}$-VIII ${ }^{\mathrm{e}}$ siècles. Elles indiquent également que ce foyer de peuplement se développe dans une zone, qui, jusque-là, était documentée par la seule présence des églises Saint-Rémy et Saint-Jean-au-Marché dont les dates de fondation demeurent incertaines ${ }^{24}$ - et par des structures de conservation et de probables habitats ${ }^{25}$. Elles démontrent enfin que ce secteur, situé hors des zones marécageuses, est très tôt occupé et rapidement intégré à l'espace urbain. Au second Moyen Âge, en effet, celui-ci devient le cœur économique de la cité ; il est englobé par la muraille défensive au XII ${ }^{\mathrm{e}}$ siècle ${ }^{26}$.

La dernière phase d'occupation date du XI ${ }^{e}$ et du début du XII siècle (fig. 5).

Fig. 5 - Troyes à la fin du haut Moyen Âge, XI siècle-début du XII ${ }^{\mathrm{e}}$ siècle (CAO C. Bourguignon, 2014 ; fond de plan : DEBORDE et LENOBLE, 1995, pl. 7).

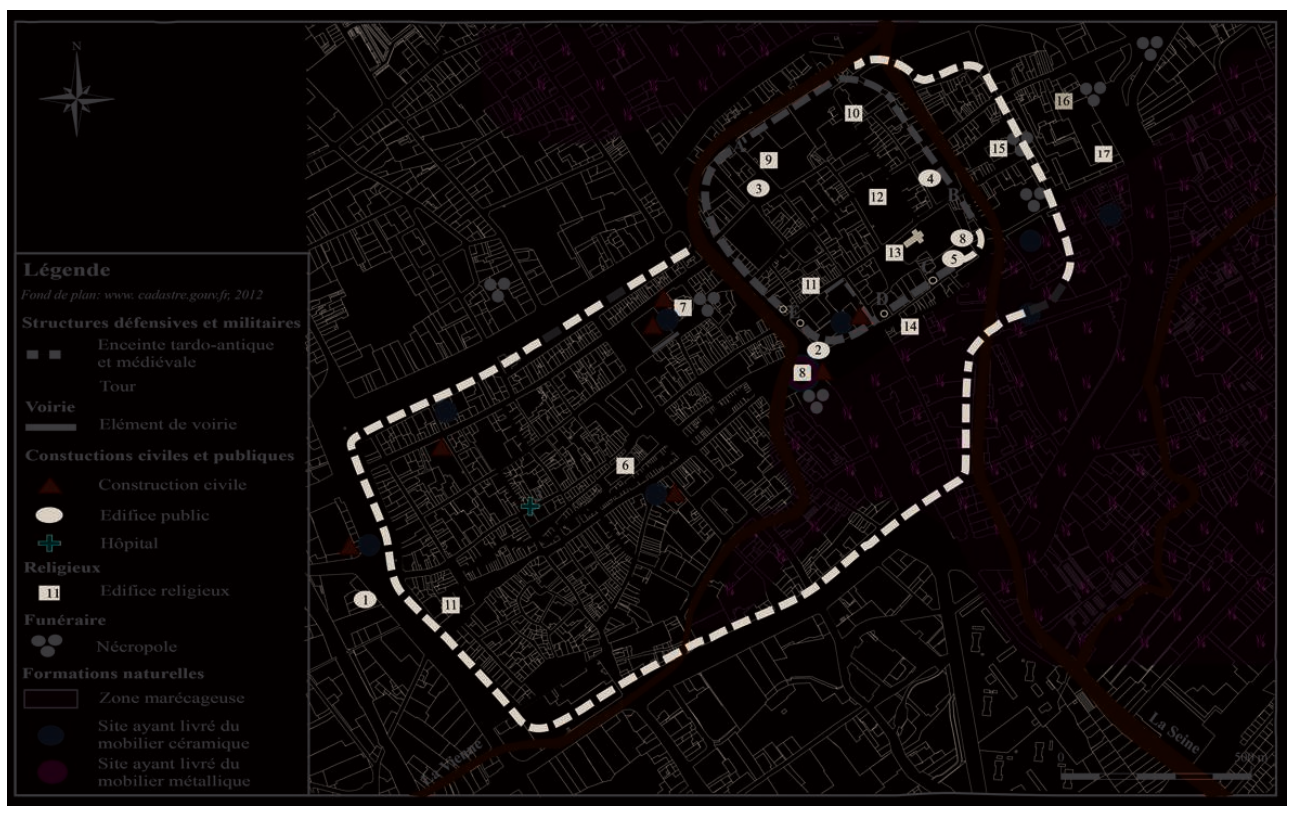

Le mobilier céramique représente l'essentiel du mobilier découvert. Il s'agit de fragments d'oules - pots à cuisson ou à stockage -, par exemple au 7, rue de l'Isle ${ }^{27}$, voire de récipients archéologiquement complets destinés au service de la table, comme la cruche à deux anses verticales à pâte blanche mise au jour place des Halles ${ }^{28}$. Un décor flammulé orne certains vases ${ }^{29}$, comme celui trouvé rue des Gayettes, qui présente des flammules de couleur rouge. Une nouvelle gamme de pots se répand donc dans la production céramique domestique à cette période : les cruches complètent les pots à cuire, les récipients à goulots apparaissent. Le décor flammulé, typique des faciès champenois des $\mathrm{XI}^{\mathrm{e}}-\mathrm{XII}^{\mathrm{e}}$ siècles, prédomine (fig. 6). 
Fig. 6 - Mobilier céramique du $\mathrm{Xl}^{\mathrm{e}}$ et début du $\mathrm{XII}^{\mathrm{e}}$ siècle mis au jour à Troyes dans l'Aube (CAO C. Bourguignon, 2014 d'après LENOBLE, 1987, ROMS, 2009 et STOCKER, 2010).

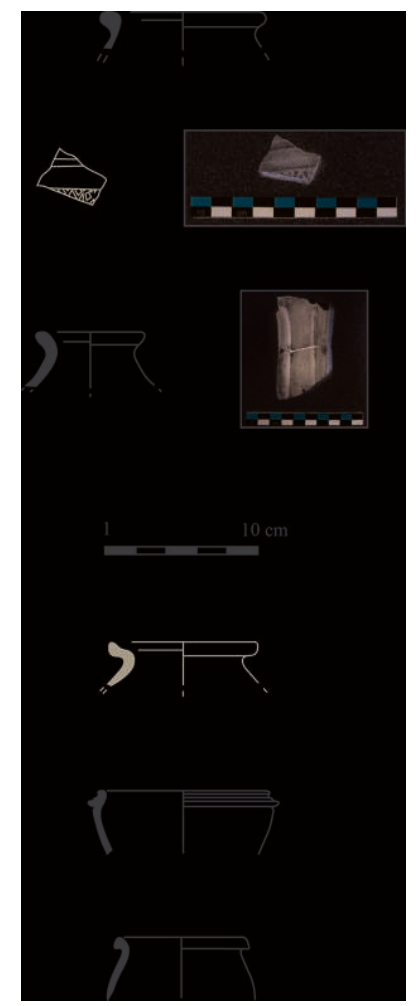

La partie sud extra-muros est la mieux renseignée par les découvertes de mobilier. Les vestiges mobiliers et structurels issus des fouilles de la place de la Libération indiquent l'existence d'activités de conservation des céréales (silos) proches de l'espace cimétérial autour de l'abbaye Notre-Dame-aux-Nonnains. Ces activités pourraient être liées à la fonction fiscale détenue par l'autorité ecclésiastique (perception de la dîme) ${ }^{30}$. L'extension de la zone funéraire pourrait être un indice de la fonction paroissiale acquise par ce cimetière. Extra-muros, à l'est, les découvertes concernent des sites occupés dès le début du haut Moyen Âge. Cependant, les contextes de ces découvertes ne permettent pas de déduire la vocation précise de ce secteur - habitat lié à la proximité de communautés monastiques? La pérennité de l'occupation est également assurée extra-muros au nord-ouest. Les vestiges découverts témoigneraient de l'existence de probables habitats liés à des structures de conservation des denrées alimentaires, caractéristiques de certains sites d'habitats ruraux du haut Moyen Âge. Les comparaisons réalisées avec le site d'habitat rural de Fontvannes/Les Tomelles, situé à une trentaine de kilomètres à l'ouest de Troyes ${ }^{31}$, concernant les structures et le mobilier - silos, puits, décor flammulé, etc. -, semblent étayer cette hypothèse.

Dans cette étude, la question de la pertinence du corpus doit être posée. Du point de vue quantitatif, il est certain que l'ensemble analysé ne peut être représentatif. Dans d'autres villes, certains sites urbains du haut Moyen Âge ont pu livrer le même nombre d'éléments que celui découvert à l'échelle de la ville de Troyes ${ }^{32}$. Cependant, les niveaux du haut Moyen Âge fournissent souvent de faibles quantités d'artefacts, ce qui nécessite de mettre en œuvre une démarche adaptée dans l'étude de la culture matérielle. Du point de vue qualitatif, les premières observations montrent que le 
mobilier troyen est assez représentatif des découvertes effectuées en milieu rural en Champagne méridionale - répertoire des formes homogène, par exemple.

Finalement, cette étude montre que les découvertes de mobilier, bien que peu nombreuses, renseignent sur les pratiques - préparations culinaires, service de la table - et les lieux de vie - proximité de l'enceinte défensive et des communautés monastiques - des populations « urbaines » du haut Moyen Âge à Troyes. Couplée à un bilan documentaire, elle précise le schéma de développement urbain. Les foyers de peuplement, localisés extra-muros dans les parties est, sud et ouest de la cité, semblent avoir abrité différentes fonctions (religieuse, funéraire), entraînant une progressive structuration de l'espace.

Ce schéma de développement urbain multipolaire est relativement classique. Diverses études d'archéologie urbaine ont en effet montré que l'éclatement des foyers de peuplement caractérise nombre de cités d'origine antique perdurant au haut Moyen Âge. Dans la métropole ecclésiastique de Tours (Indre-et-Loire), un bourg monastique se développe dès la fin du $v^{e}$ siècle extra-muros, à l'ouest, autour de la basilique SaintMartin et de ses satellites. Aux XI $\mathrm{e}^{\mathrm{e}} \mathrm{XI}{ }^{\mathrm{e}}$ siècles, c'est une véritable agglomération tournée vers le fleuve, caractérisée par un réseau viaire dense, qui jouxte la cité d'origine antique désormais fortifiée ${ }^{33}$. À Chartres (Eure-et-Loir), plusieurs foyers de peuplement, matérialisés par des constructions sur poteaux, des cabanes excavées, des fosses et des inhumations sont attestés en contrebas du promontoire, sur lequel s'est développée la ville antique encore occupée au haut Moyen Âge ${ }^{34}$. Près de Troyes, la ville d'Auxerre (Yonne) connaît également un développement polynucléaire autour de fondations religieuses aux $\mathrm{V}^{\mathrm{e}}-\mathrm{XI}^{\mathrm{e}}$ siècles et le long d'axes de circulation et de voies d'eau dès la fin du haut Moyen Âge ${ }^{35}$.

L'étude montre ainsi que le mobilier troyen du haut Moyen Âge constitue un potentiel informatif intéressant pour l'étude de la ville médiévale, qui ne peut être réduite à « un corps étranger, un kyste, une malformation dans la société médiévale ${ }^{36}$ ".

\section{NOTES}

1. H. GALINIÉ, «L'entre-deux : les terres noires des cités ", in B. BEAUJARD (dir.), La naissance de la ville chrétienne, mélanges en hommages à Nancy Gauthier, t. 1, Tours, 2002, p. 97-106; H. GALINIÉ, « La question urbaine entre Antiquité et Moyen Âge: "l'entre-deux des cités" (250-950) ", in J. CHAPELOT (dir.), Trente ans d'archéologie médiévale en France. Un bilan pour un avenir, Caen, 2010, p. 337-350 ; E. LORANS, A.-M. JOUQUAND et al., Les rythmes de l'espace urbain à Tours : nouvelles données, nouvelles questions ( $\mathrm{I}^{e r}-\mathrm{X}^{e}$ siècle apr. J.-C.), in E. LORANS et X. RODIER (dir.), Archéologie de l'espace urbain, Tours, 2013, p. 209-220.

2. C. BouRGUIGNON, Évolution de la topographie urbaine de la ville de Troyes du Haut-Empire au début du second Moyen Âge (I $I^{e r}$-XII $e^{e}$ siècle). Essai de synthèse des données archéologiques et historiques, 2 vol., mémoire de master 1, sous la direction de S. Wirth, D. Cailleaux, université de Bourgogne et M. Kasprzyk, Inrap, Dijon, 2012. 
3. C. B OURGUIGNON, Approche des modalités d'occupation du sol d'espaces urbains et ruraux sur le territoire de la ville et du diocèse de Troyes (Aube) à partir d'un corpus de mobilier archéologique altomédiéval, 2 vol., mémoire de master 2, sous la direction de S. Wirth, D. Cailleaux, université de Bourgogne et M. Kasprzyk, Inrap, Dijon, 2013.

4. Il s'agit ici principalement du mobilier céramique qui a été réexaminé dans le cadre d'un Projet d'Action Scientifique portant sur l'habitat rural du haut Moyen Âge en ChampagneArdenne, dirigé par M.-C. Truc et A. Rémy (Inrap).

5. L. PIETRI, «Troyes ", in N. GAUTHIER et J.-C. PICARD (dir.), Topographie chrétienne des cités de la Gaule des origines au milieu du VIII siècle, t. 7, Paris, 1994, p. 67-80 ; M. LENOBLE et J. DEBORDE, Troyes. Document d'évaluation du potentiel archéologique urbain, Tours, 1995 ; I. CRÉTÉ-PROTIN, Église et vie chrétienne dans le diocèse de Troyes (IVe-IXe siècle), Villeneuve-d'Ascq, 2002, publication remaniée d'une thèse de doctorat d'histoire médiévale soutenue à l'université de Paris IV Sorbonne sous la direction de M. Rouche.

6. J.-C. COURTALON-DelaistRE, Topographie historique de la ville et du diocèse de Troyes, Troyes, 1783.

7. T. Bоutiot, Histoire de la ville de Troyes et de la Champagne méridionale, t. 1, Bruxelles, 1870.

8. T. BOUTIOT et E. SOCARD, Dictionnaire topographique du département de l'Aube, Paris, 1874.

9. J. Lusse et A. PATRolin (dir.), «L'archéologie en Champagne-Ardenne (1960-1990) », Bulletin de la Société archéologique champenoise, 85/4 (1992), p. 3-422.

10. M. LeNOBLE et J. DeBORDE, Document..., op. cit.

11. I. CRÉTÉ-PROTIN, Église et vie chrétienne..., op. cit.

12. B. F ORT et N.T ISSERAND (dir.), «Le mobilier métallique et l' Instrumentum: approches méthodologiques ", Les nouvelles de l'archéologie, 131 (2013), p. 3-62.

13. P. S TOCKER, Troyes. 3-5, Rue du Bon Pasteur. Rapport de diagnostic archéologique, DRAC/SRA Champagne-Ardenne, Châlons-en-Champagne, 2010.

14. G. DEBORDE, Troyes. Rue des Guillemets/Rue Breslay. Rapport de diagnostic archéologique, DRAC/SRA Champagne-Ardenne, Châlons-en-Champagne, 2010.

15. I. CRÉTÉ-Protin, Église et vie chrétienne..., op. cit., p. 203, 205 et 208.

16. M. LENOBLE et J. DEBORDE, Document..., op. cit., p. 34-35.

17. G. DEBORDE, Troyes, Institut universitaire des métiers, Rapport final d'opération, 1992, DRAC/SRA Champagne-Ardenne, Châlons-en-Champagne, 2006 ; J. BIEnAIMÉ, Troyes, Maison des jeunes et de la culture. Rapport de sauvetage urgent, DRAC/SRA Champagne-Ardenne, Châlons-en-Champagne, 1964.

18. A. Louis, Troyes, 76-78, Mail des Charmilles. Sépultures du Bas-Empire en bordure du decumanus d'Augustobona. Rapport de diagnostic archéologique, DRAC/SRA Champagne-Ardenne, Châlons-enChampagne, 2011.

19. P. KuCHLER et C. Roms (dir.), Troyes. Place de la Libération. Formation et développement d'un espace urbain de l'Antiquité à nos jours. Rapport final d'opération, DRAC/SRA Champagne-Ardenne, Châlonsen-Champagne, 2011.

20. M. L ENOBLE (dir.), Troyes. Place des Halles. Rapport de sauvetage programmé, DRAC/SRA Champagne-Ardenne, Châlons-en-Champagne, 1987.

21. J. SCAPULA, «Les récentes découvertes archéologiques de Troyes : Collège Saint-Bernard », La vie en Champagne, 137 (1965), p. 14-15.

22. C. R oms, Troyes. Rue du Général de Gaulle/Rue Argence. Rapport de diagnostic, DRAC/SRA Champagne-Ardenne, Châlons-en-Champagne, 2009.

23. J. SCAPULA, «Les nouvelles découvertes archéologiques de Troyes : Place du Général Patton/ Rue Voltaire ", La vie en Champagne, 105 (1962), p. 11-12.

24. A. ROSEROT, Dictionnaire historique de la Champagne méridionale (Aube), des origines jusqu'à 1790, avec une introduction sur l'histoire de cette région, Troyes, 1948, t. 3, p. 1611 ; I. CRÉTÉ-PROTIN, Église et vie chrétienne..., op. cit., p. 325. 
25. M. LENOBLE et J. DEBORDE, Document..., op. cit., p. 35.

26. B. COLLET, Troyes et ses fortifications. Tours, portes, arches, Troyes, 1988, p. 7.

27. A. Louis et C. Roms, Un quartier artisanal médiéval et moderne : la fouille du «Campus universitaire de centre-ville» et de la "Résidence de l'Isle» à Troyes. Rapport final d'opération de fouilles archéologiques, DRAC/SRA Champagne-Ardenne, Châlons-en-Champagne, 2009.

28. M. Lenoble (dir.), Troyes. Place des Halles..., op. cit., p. 37, fig. 38, $\mathrm{n}^{\circ} 2$.

29. Un décor flammulé est composé de bandes obliques ou verticales, disposées par groupe de trois à cinq, réalisées à l'aide de coulures de peinture de couleur ocre à marron.

30. P. KuCHLER et C. Roms (dir.), Troyes. Place de la Libération... op. cit., p. 536.

31. Le mobilier céramique trouvé à Fontvannes/Les Tomelles, lors des fouilles réalisées au début des années 1990, a fait l'objet d'une nouvelle étude dans le cadre du master 2. Le corpus était composé de 2387 tessons.

32. À Reims (Marne), le site du 19, rue Eugène Desteuque, fouillé en 2004, a livré 732 tessons attribuables aux productions du haut Moyen Âge. S. SinDonino, Reims. 19, Rue Eugène Desteuque. Rapport final d'opération, DRAC/SRA Champagne-Ardenne, Châlons-en-Champagne, 2005.

33. H. GaliniÉ, « Tours de Grégoire, Tours des archives du sol », in N. GAUTHIER et H. Galinié (dir.), Grégoire de Tours et l'espace gaulois, Tours, 1997, p. 65-80 ; H. GALINIÉ, Tours antique et médiéval. Lieux de vie, temps de la ville: 40 ans d'archéologie urbaine, Tours, 2007 ; H. NOIZET, La fabrique de la ville. Espaces et sociétés à Tours (IX'-XIII ${ }^{e}$ siècle), Paris, 2007.

34. C. Ben KadDour, D. Joly et S. WilleRval, « Chartres et sa proche campagne au haut Moyen Âge (fin $\mathrm{v}^{\mathrm{e}}$-fin $\mathrm{x}^{\mathrm{e}}$ siècle). Topographie urbaine et péri-urbaine, analyse de structures et étude du mobilier : un premier bilan ", Revue archéologique du Centre de la France, 53 (2014) [mis en ligne le 15 avril 2015].

35. C. S APIN (dir.), Auxerre. Document d'évaluation du potentiel archéologique urbain, Tours, 1998, p. 35-37 et 42-43.

36. R. FossIER, Enfance de l'Europe ( $X^{e}$-XII $e^{e}$ siècle), t. 1, Paris, 1982, p. 980.

\section{INDEX}

Mots-clés: mobilier céramique, mobilier métallique, occupation du sol, topographie urbaine, habitat rural, diocèse de Troyes, haut Moyen Âge

Geographical index: France/Troyes

\section{AUTHOR}

\section{CLAIRE BOURGUIGNON}

Diplômée d'un master 2 recherche Archéologie, cultures, territoires, environnement - Université de Bourgogne/université de Franche-Comté 